\section{Investigación clínica de la ptosis cerebral. Presentación de seis nuevos casos}

\author{
LUIS CARTIER R., JORGE PARRA, JUAN P. CONTRERAS S.
}

\section{Cerebral ptosis. Analysis of six cases}

\begin{abstract}
Background: Cerebral ptosis is understood as the bilateral paralysis of eyelid elevation linked to a stroke or hemorrhage of the middle cerebral artery (MCA). It is a transient condition, independent of the evolution of the lesion. Aim: To analyze six patients with the condition. Patients and Methods: Report of five women and one male aged 42 to 72 years. Results: All suffered an infarction or hemorrhage in the territory of the middle cerebral artery of the non-dominant hemisphere and developed a bilateral palpebral ptosis. The recovery started after the fourth day. At the tenth day, eye opening was effortless and did not require frontal help, despite the persistence of hemiplegia. Conclusions: Cerebral ptosis is a mimetic dysfunction of a specific non-injured area of the cerebral cortex, originated from a nearby parenchymal damage such as the middle cerebral artery of the same hemisphere. Cerebral ptosis expresses the inhibition of the voluntary eyelid elevation center, of prefrontal location in the non-dominant hemisphere.

(Rev Med Chile 2018; 146: 857-863)
\end{abstract}

Key words: Blepharoptosis; Middle Cerebral Artery; Stroke.

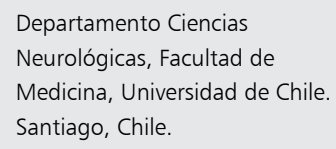

Los autores declaran no tener conflictos de interés.

Trabajo no recibió

financiamiento.

Recibido el 5 de enero de 2018 , aceptado el 20 de julio de 2018.

Correspondencia a :

Dr. Luis Cartier Rovirosa Departamento Ciencias Neurológicas, Facultad de Medicina, Universidad de Chile. Santiago, Chile.

Icartier@med.uchile.cl
So e ha identificado como "ptosis cerebral" (PC) la parálisis de la elevación voluntaria de los parpados, en el contexto de infartos o hemorragias del territorio de la arteria cerebral media, en ausencia de daño mesencefálico, de nervios oculomotores, del simpático cervical o de la musculatura extraocular ${ }^{1}$.

Caplan, en 1974, en su trabajo sobre ptosis, admitió que fue Best en 1931 quien identificó la PC 2 . Manconi y colaboradores revisaron 75 casos de PC publicados hasta el año 2006, estableciendo que la parálisis de la elevación voluntaria de los párpados, o blefarocolisis, estaba vinculada a lesiones de la arteria cerebral media (ACM) unilaterales, donde $87,8 \%$ de casos correspondían a infartos y $12,2 \%$ a hemorragias y mayoritariamente coincidían con el compromiso del hemisferio derecho ${ }^{3}$, esta susceptibilidad del hemisferio no dominante ya había sido observada ${ }^{4}$.

También puede verse afectada la apertura voluntaria de los ojos con el blefaroespasmo, oclusión involuntaria y súbita de los párpados, seguida de una breve incapacidad para lograr la apertura palpebral, fenómeno diskinético cortico-subcortical ${ }^{5}$; asimismo, la incapacidad para controlar la apertura voluntaria de los ojos o apraxia de la apertura palpebral, por disfunción cortical ${ }^{6}$. Estas alteraciones podrían confundirse con la PC, pudiendo ser necesario un estudio electromiográfico con registro simultáneo del orbicular y elevador de los párpados para su identificación. El blefaroespasmo muestra actividad del orbicular y ausencia de respuesta del elevador, en la apraxia se observa actividad eléctrica variable de ambos músculos y en la $\mathrm{PC}$ reina el silencio eléctrico ${ }^{7}$.

El seguimiento de seis nuevos casos de ptosis cerebral, nos ha permitido un análisis complementario de esta patología que afecta la elevación palpebral, obteniendo información significativa para su interpretación patogénica. 


\section{Pacientes}

\section{Caso 1}

Mujer de 72 años, diestra, hipertensa, diabética, consultó por súbito compromiso de conciencia y déficit motor de las extremidades izquierdas. Ingresó en vigilia, desorientada, con hemiplejia, hipoestesia y hemianopsia izquierdas, con comunicación verbal conservada. La tomografía computada (TC) cerebral mostró infarto del territorio de la cerebral media derecha (Figura 1). Se sospechó embolia pulmonar en la radiografía de tórax, cardiomegalia y congestión pulmonar, que obligó su hospitalización en cuidados intensivos (UCI). El control neurológico del día siguiente identificó una ptosis palpebral en ausencia de compromiso de la oculomotilidad voluntaria o refleja y la hemiplejia izquierda. Al cuarto día había comenzado a entreabrir los párpados con ayuda frontal, la apertura palpebral que lograba no se mantenía, cerrándosele rápidamente los ojos. Obtuvo mayor apertura de los ojos desde el quinto día y se mantenían abiertos más tiempo. Después del décimo día, la apertura ocular se hizo completa y duradera, logrando abrir completamente los ojos sin esfuerzos.

\section{Caso 2}

Mujer de 42 años, diestra, ingresó por intensa cefalea, debilidad de extremidades izquierdas y pérdida del control esfinteriano. Bien orientada, era capaz de invertir series automáticas. Hemiplejia e hemihipoestesia izquierdas de predominio faciobraquial, dificultad para elevar los párpados; la apertura mecánica de los ojos evidenció pupilas iguales, reactivas, con oculomotilidad voluntaria y refleja conservadas, mantenía desviación conjugada de la mirada a derecha. TC mostró una hipodensidad heterogénea en región fronto-témporo-parietal derecha y ausencia del llene de la arteria carótida derecha (Figura 1). Con diagnóstico de accidente cerebrovascular maligno, se realizó craneotomía descompresiva. Conservó la vigilancia en la etapa postoperatoria inmediata, así como la hemiplejia izquierda y la ptosis palpebral. Al cuarto día, el ojo izquierdo (lado de la plejia) inició semiapertura palpebral, mientras la ptosis derecha persistía. Al quinto día se inició apertura ocular derecha, elevación parcial y transitoria del párpado con apoyo frontal, el ojo izquierdo había completado su apertura. Al noveno día se comprobó la apertura voluntaria sin esfuerzos de ambos ojos.

\section{Caso 3}

Hombre de 66 años, zurdo, hipertenso, despertó desorientado y disártrico, con déficit sensitivo-motor de extremidades derechas. Ingresó en vigilia, cumplía órdenes simples, sin embargo, mantenía cerrados los ojos, la apertura mecánica de los párpados mostró pupilas iguales, oculomotilidad conservada con mirada preferencial a izquierda, hemiplejia faciobraquiocrural derecha y hemianopsia. La TC de cerebro mostró hematoma fronto-parietal izquierdo (Figura 2). Al segundo día, debido al progresivo deterioro clínico, fue necesario drenar el hematoma, que evolucionó sin complicaciones. Permaneció comprometido de conciencia hasta el sexto día. Cuando recuperó la vigilancia podía apretar la mano izquierda, pero era incapaz de abrir voluntariamente los ojos, se pudo comprobar que conservaba la oculomotilidad voluntaria y dirigía la mirada a la orden. Desde el octavo día inició apertura palpebral parcial y transitoria del ojo izquierdo. En los días subsiguientes lograba la elevación transitoria de ambos párpados, notándose al decimosegundo día mayor apertura del ojo derecho. Después del decimotercer día tenía elevación persistente de ambos párpados.

\section{Caso 4}

Mujer de 70 años, diestra, ingresó por cefalea intensa y vómitos, debilidad de hemicuerpo izquierdo y disartria. En vigilia, orientada, inatenta, hemiplejia faciobraquiocrural (FBC) izquierda e incapacidad para abrir los ojos. La TC de cerebro mostró lesión hipodensa extensa en territorio de la arteria cerebral media izquierda, con pequeño foco hemorrágico (Figura 2). Presentó neumonía intrahospitalaria, resuelta con tratamiento antibiótico. Durante este período, la PC se mantuvo, sin compromiso de la oculomotilidad. Al sexto día inició a entreabrir los párpados con ayuda frontal. $\mathrm{Al}$ octavo, noveno día logró mantener apertura parcial de los párpados, solo abrió completamente los ojos al décimo día y al decimosegundo sin ayuda frontal.

\section{Caso 5}

Mujer de 64 años, diestra, dislipidémica y tabáquica. Ingresó desorientada, disártrica, con 


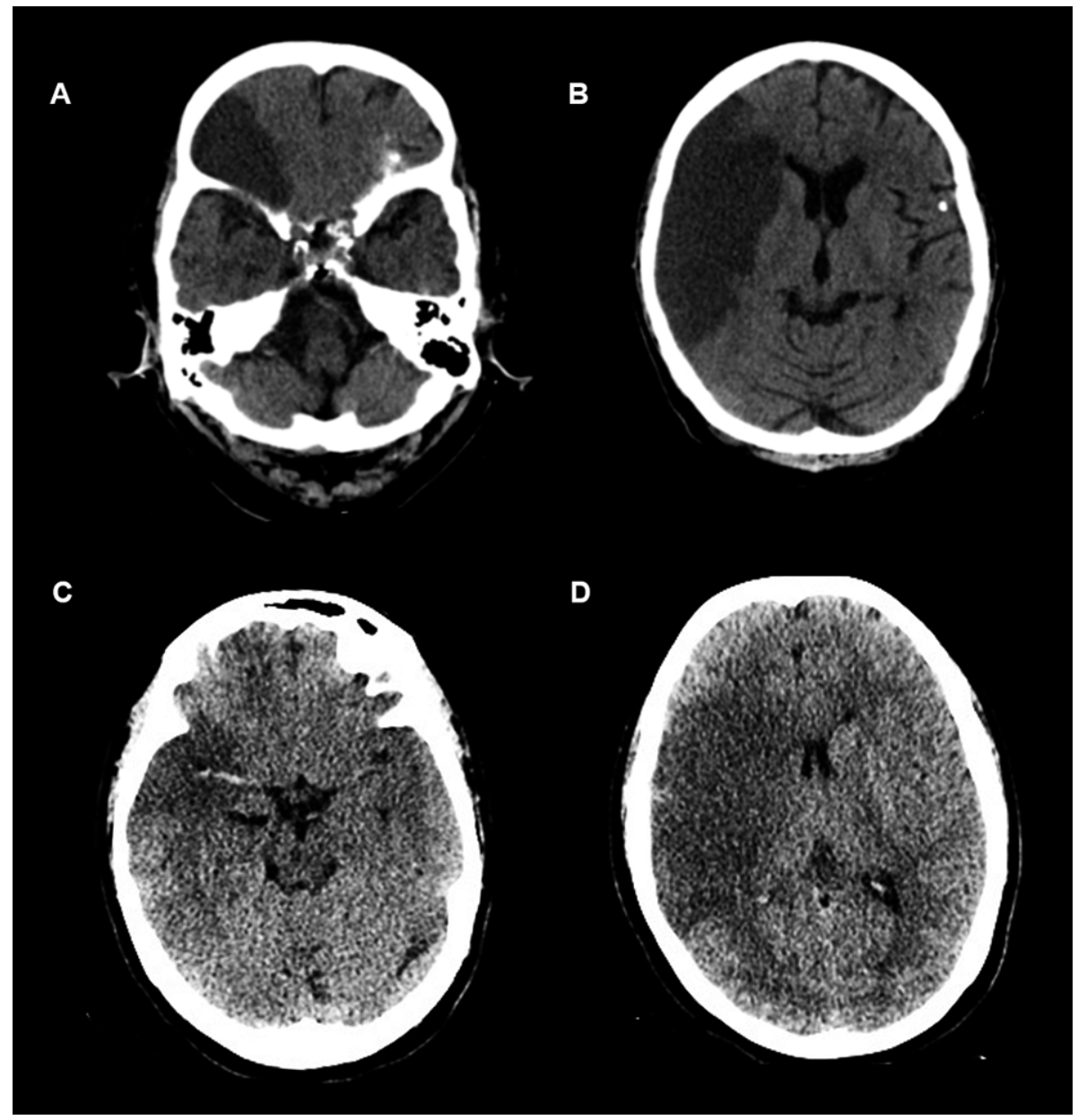

Figura 1. TC cerebro sin contraste, cortes axiales, caso $\mathrm{N}^{\circ} 1(\mathbf{A}, \mathbf{B})$ y caso $N^{\circ} 2(\mathbf{C}, \mathbf{D})$.

hemiplejia izquierda. La TC cerebral mostró el signo de la cuerda en ACM derecha, pérdida de la diferenciación córtico-subcortical en el hemisferio derecho. Era incapaz de abrir los ojos a la orden, aún con esfuerzo frontal. La oculomotilidad voluntaria y refleja estaba conservada. Manifestaba intensa cefalea, el control imagenológico del tercer día mostró un infarto que comprometía todo el territorio de la arteria cerebral media del lado derecho (Figura 3). Según los especialistas no tenía indicación neuroquirúrgica. Se trasladó a la unidad de pacientes críticos con sedación y monitoreo invasivo con captor de presión intracraneana, retirado después del tercer día. Cuando readquirió vigilancia elevaba los párpados de manera incompleta y transitoria, mejoró poco a poco la persistencia de la apertura. Extubada, siguió mejorando desde el punto de vista general, logrando apertura ocular completa sin esfuerzos después del día decimotercero.

\section{Caso 6}

Mujer de 62 años, diestra, hipertensa y tabáquica que consultó por disartria y debilidad del hemicuerpo izquierdo de $2 \mathrm{~h}$ de evolución. Ingresó con un índice NIHSS (National Institute of Health Stroke Scale), con TC cerebral normal. Se indicó trombolisis endovenosa, suspendida por epistaxis. La inmediata AngioTAC de cerebro permitió definir oclusión en el segmento M1 de la ACM derecha (Figura 3). Se procedió a trombectomía mecánica logrando una revas- 


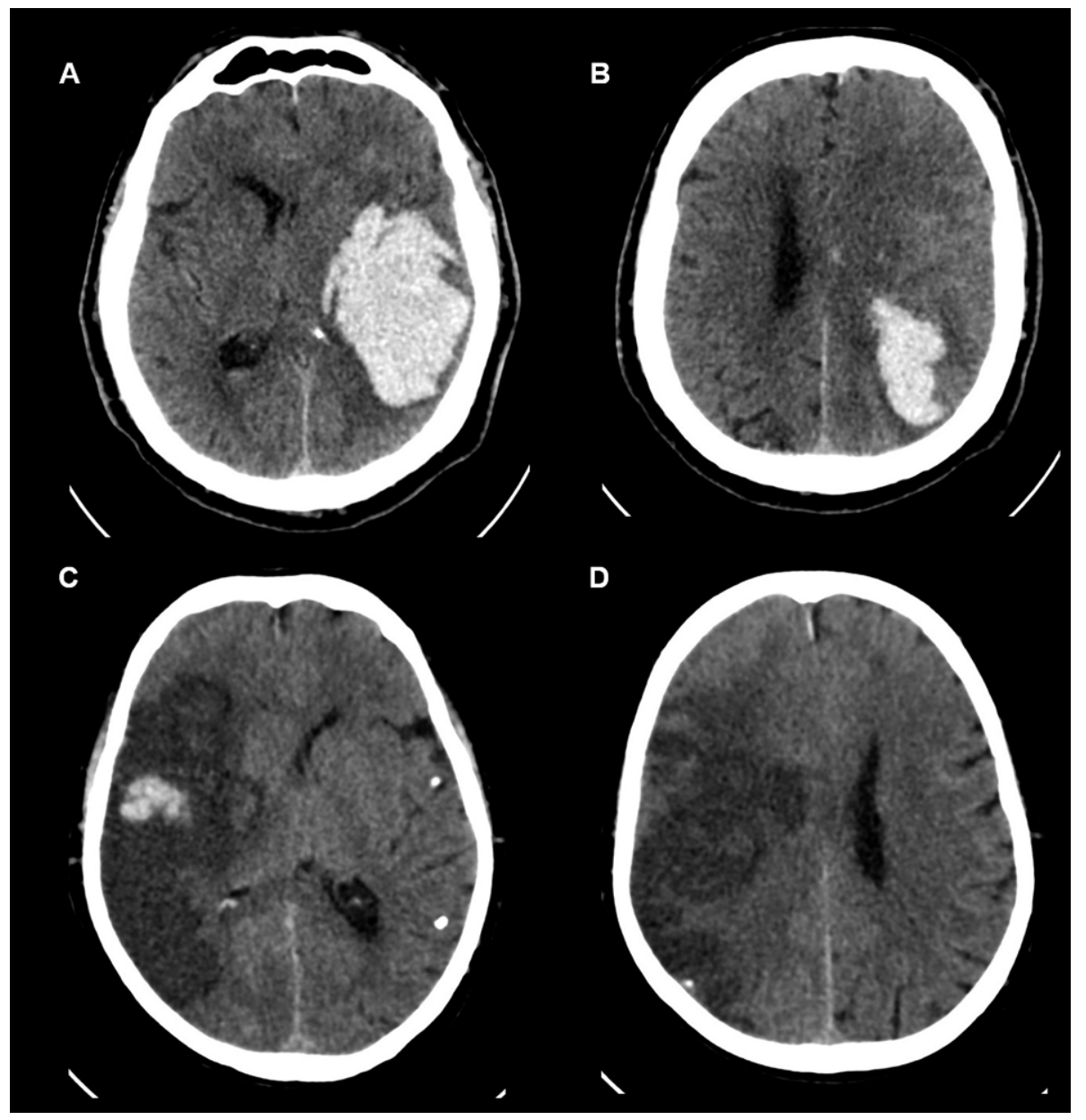

Figura 2. TC cerebro sin contraste, cortes axiales, caso $\mathrm{N}^{\circ} 3(\mathbf{A}, \mathbf{B})$ y caso $N^{\circ} 4(\mathbf{C}, \mathbf{D})$.

cularización satisfactoria (TICI 3). Al tercer día mantenía los ojos cerrados, aparentemente dormida, estimulada intenta abrir los ojos sin lograrlo. Tenía preservado el lenguaje y estaba orientada. La elevación mecánica de los párpados mostró oculomotilidad voluntaria y refleja normales. Al día siguiente se observó apertura ocular impersistente y asimétrica, ptosis mayor a izquierda. La PC se recuperó al noveno día con apertura completa y persistente.

\section{Comentario}

Se han presentado seis pacientes que, en relación con la etapa aguda de un accidente vascular del territorio de la arteria cerebral media del he- misferio no dominante (cinco infartos a derecha y una hemorragia a izquierda), presentaron una blefaroptosis bilateral de rápida recuperación. La elevación parcial y transitoria de los párpados se activó alrededor del quinto día y llegó a una apertura completa y persistente después del décimo día.

Caplan, en 1974, buscó y estudió las distintas formas de ptosis entre 284 pacientes portadores de accidentes cerebrovasculares. Logró seleccionar 25 enfermos que mostraron algún trastorno de la apertura ocular, 10 de los cuales se habían originado por lesiones mesencefálicas, en 2 formaba parte de un síndrome de Bernard-Horner y en 13 era consecuencia de lesiones en uno de los hemisferios cerebrales. Caplan identificó este último grupo como "ptosis cerebrales", encontró 


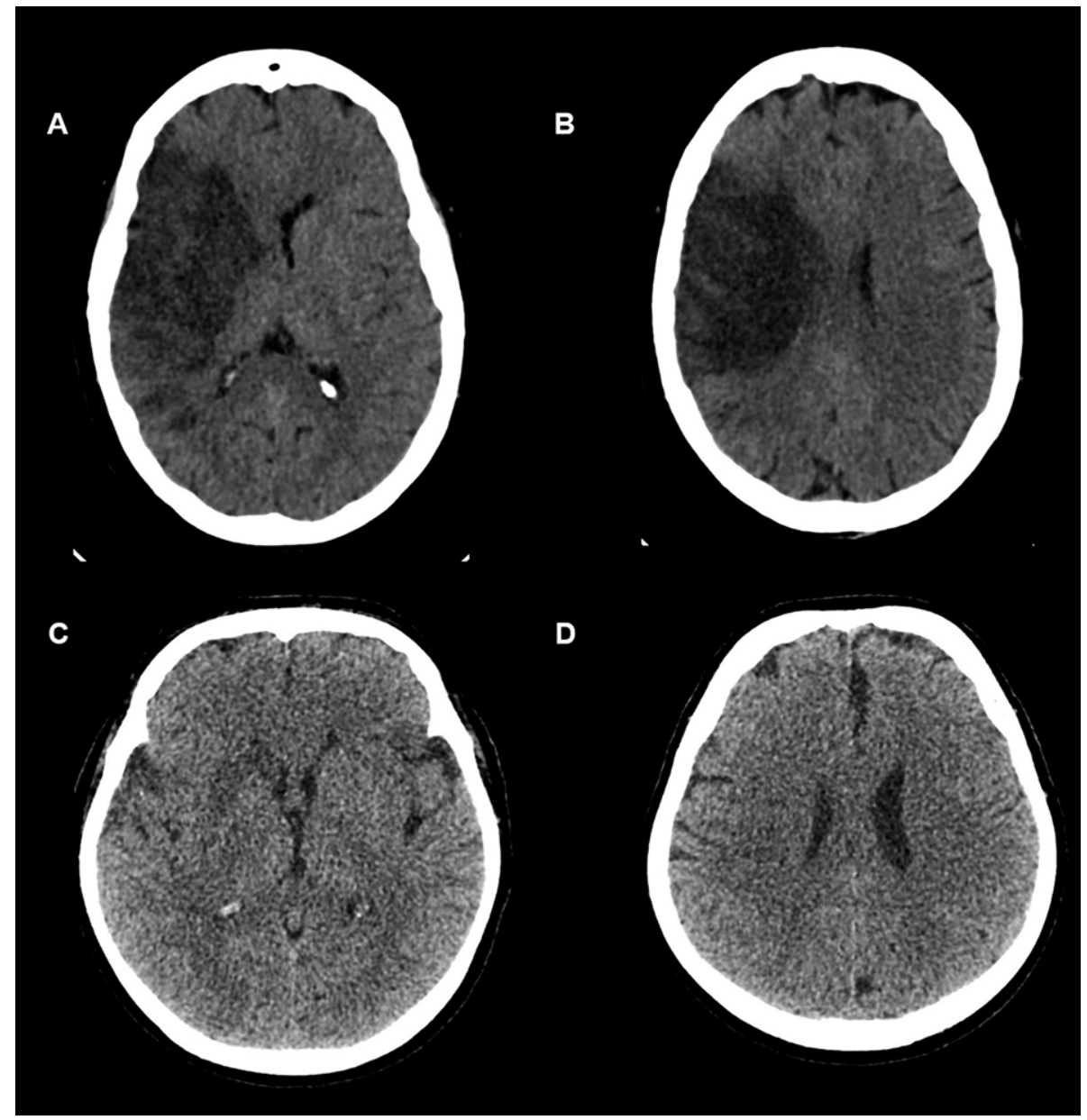

Figura 3. TC cerebro sin contraste, cortes axiales, caso $\mathrm{N}^{\circ} 5(\mathbf{A}, \mathbf{B})$ y caso $N^{\circ} 6(\mathbf{C}, \mathbf{D})$.

que la parálisis de la elevación era unilateral en 8 de los enfermos y en 5 la ptosis era bilateral, producto de una sola lesión hemisférica. Caplan, para explicar esta parálisis bilateral de la elevación originada por una lesión unilateral, recurrió a los estudios de Leyton y Sherrington (1917) y Penfield y Rasmussen (1968), quienes habían demostrado experimentalmente en monos y en el hombre que la estimulación de la corteza premotora del hemisferio derecho producía la apertura natural de ambos ojos ${ }^{2}$.

A pesar de la clarividente hipótesis de Caplan, la fisiopatogenia de la ptosis cerebral ha recibido distintas interpretaciones, como que la bilateralidad de la ptosis se debería a la existencia de un núcleo único mesencefálico para los elevadores, postulado discordante con la realidad anatómica, que ha establecido que las neuronas son específicas e inervan independientemente cada músculo elevador, (son dos núcleos en uno $)^{8}$. También se ha afirmado que, debido al edema producido por estas grandes lesiones, la ptosis bilateral tendría su origen en la compresión del tronco cerebral ${ }^{9}$, compromiso que se descartada por la ausencia de alteraciones de la oculomotilidad, la transitoriedad del fenómeno, el inicio casi inmediato de la ptosis (no espera al edema) y la recuperación sin secuelas.

Es interesante constatar que dentro del escaso número de pacientes comunicados con ptosis cerebral, mayoritariamente mostraron lesiones del hemisferio derecho ${ }^{1,3,4,10}$, habitualmente el hemisferio no dominante. Por ello, una ptosis bilateral por lesión en el hemisferio izquierdo ad- 
quiere significado. Apoyados en la sistematización desarrollada por Mac Manus, quien demostró que la zurdera está presente en $12 \%$ de los hombres $\mathrm{y}$ en $8 \%$ de las mujeres, haciendo comprensible que $10 \%$ de la humanidad tenga como hemisferio no dominante el hemisferio izquierdo, condición fisiológica que implica la zurdera ${ }^{11}$. Eventuales lesiones de zurdos en el hemisferio izquierdo (no dominante) permiten expresar en ellos ptosis cerebral. Es demasiado significativa la preferencia de la ptosis cerebral como manifestación del hemisferio no dominante para soslayarla, hecho que sugiere la existencia de un centro que controla la apertura voluntaria de los párpados en áreas prefrontales del mismo.

La serie de Averbuch-Heller y cols. ${ }^{10}$, y la nuestra, confirman que la ptosis cerebral se vincula con el hemisferio no dominante. Averbuch-Heller y colaboradores estudiaron 64 pacientes, entre los cuales, 24 presentaron algún compromiso en la apertura voluntaria de los ojos, que en 14 fue unilateral y en 10 bilateral configurando una ptosis cerebral. En 8 de estos 10 pacientes, la lesión fue del hemisferio derecho, no se especifica si los que tenían lesión en el hemisferio izquierdo eran zurdos. Sin embargo, por estadísticas al menos uno debería ser zurdo. Proporción concordante con lo encontrado en nuestra serie, donde 5 de 6 tenían lesión en el hemisferio derecho, pero los seis tenían lesionado el hemisferio no dominante, porque el paciente de la lesión izquierda era zurdo.

La semejanza en tiempo y modo de evolución de los casos de PC demuestran su carácter funcional y que esta área disfuncional está fuera del territorio lesionado de la ACM. Estableciendo que la PC es la expresión de una diasquisis; alteración que se define como la disfunción mimética y transitoria de un área no lesionada de la corteza cerebral, relacionada con un daño en etapa aguda del mismo hemisferio. La ptosis cerebral se origina por el daño al territorio de la arteria cerebral media del hemisferio no dominante, génesis de la inhibición del centro de la apertura voluntaria de los ojos.

La hipótesis funcional parece convalidarse al analizar a la enferma que inició precozmente la reapertura ocular en el lado hemipléjico. Pudo interpretarse como una disminución del tono muscular por aumento de la complacencia de ese orbicular pléjico, como les pareció a algunos autores con anterioridad ${ }^{10}$, sin embargo, ese hecho puso en evidencia que la desaparición precoz de la ptosis en el lado pléjico sugería una atenuación o pérdida de la inhibición paralizadora en ese lado. Se había inhibido la diasquisis, probablemente debido a un aumento del edema postlesional que no acentuó la diasquisis, sino que originó su precoz desaparición. La asimetría que en general se observa en la recuperación de la ptosis ha permitido confirmar la controvertida independencia de los músculos elevadores, tanto en su inervación como de su representación cortical, demostrando también, la frecuente intervención primaria de la corteza cerebral en actividades automáticas, como la hiperapertura ocular en la sorpresa, el control de la mirada y de la apertura ocular voluntaria.

Las grandes lesiones hemisféricas suelen mostrar miradas preferenciales hacia el lado de la lesión o parálisis de la mirada hacia el lado hemipléjico. Esta desviación de los ojos y la cabeza solo se observa en la etapa aguda de las grandes lesiones hemisféricas, semejante a la ptosis cerebral, sugiriendo que la desviación conjugada de la mirada también podría corresponder a una diasquisis. Aunque la interpretación más corriente de la desviación de la mirada se correlaciona con la lesión de fibras motoras que interviene en la mirada voluntaria, también se ha interpretado como una alteración práxica $y$, más consistentemente, como una negligencia atribuible a la lesión del hemisferio derecho ${ }^{12}$, poniendo poca atención a la transitoriedad del fenómeno, que generalmente permite descartar lo lesional. Los estudios de Singuer y colaboradores buscaron correlacionar la desviación de la mirada con la gravedad de la lesión. En un estudio de 116 enfermos, el 20\% de los más graves presentaba desviación de la mirada, curiosamente esta condición predominó significativamente en lesiones del hemisferio derecho ${ }^{13}$. En nuestra corta serie de grandes lesiones de la ACM relacionadas a blefaroptosis, la desviación de la mirada se pesquisó en $2 / 6,30 \%$ de un grupo de pacientes particularmente graves, donde la desviación de la mirada duró lo mismo o menos que la ptosis.

En conclusión, la ptosis cerebral y, eventualmente, la desviación conjugada de la mirada habla de una diasquisis prefrontal, originada en una lesión del territorio de la cerebral media del hemisferio no dominante. 


\section{Referencias}

1. Krohel GB, Griffin JF. Cortical blepharoptosis. J Am Ophthalmol 1978; 85: 632-4.

2. Caplan LR. Ptosis. J Neurol Neurosurg Psychiatry 1974; 37: 1-7.

3. Manconi M, Cesnik E, Casetta I, Govoni V, Ferini l, Granieri E. Cerebral blepharoptosis: two new cases, literature review for diagnostic criteria. Neurol Sci 2006; 27: 161-5.

4. Averbuch-Heller 1, Stahl JS, Remler BF, Leigh RJ. Bilateral ptosis and upgaze palsy with right hemispheric lesions. Ann Neurol 1996; 40: 465-8.

5. Anderson RL, Digre KB. Apraxia of lid opening in blepharospasm. Ophthalmic surg 1990; 21 (5): 331-4.

6. Lepore FE, Duvoisin RC. "Apraxia” of eyelid opening: an involuntary elevator inhibition. Neurology 1985; 35 : 423-7.

7. Aramideh M, Ongerboer de Visser B, Koelman JH, Bour LJ, Devriese PP, Speelman JD. Clinical and electromyographic features of levator palpebrae superioris muscle dysfunction in involuntary eyelid closure. Mov Disord 1994; 9: 395-402.

8. Esteban A, Traba A, Prieto J. Eyelid movements in health and disease. The supranuclear impairment of the palpebral motility. Neurophysiol Clin 2004; 34: 3-15

9. Blacker DJ, Wijdicks EF. Delayed complete bilateral ptosis associated with massive infarction of the right hemisphere. Mayo Clin Proc 2003; 78: 836-9.

10. Averbuch-Heller L, Leigh RJ, Mermelstein V, Zagalski L, Streifler Y. Ptosis in patients with hemispheric strokes. Neurology 2002; 58: 620-4.

11. Mcmanus I. The history and geography of human handedness. En: Sommer IEC, Kahn RS, editores, Language lateralization and psychosis. Cambridge University Press. Cambridge, Reino Unido. 2009.

12. Berger MF, Ilg U, Karnath H. Deviation of eyes and head in acute cerebral stroke. BMC Neurology 2006; 6, 23: 3-8.

13. Singer OC, Marek C, Humpich MC, Laufs H, Lanfermann $\mathrm{H}$, Steinmetz $\mathrm{H}$, et al. Conjugate eye deviation in acute stroke incidence, hemispheric asymmetry, and lesion pattern. Stroke 2006; 37: 2726-32. 